\title{
Immunohistochemical expression levels of p53 and eIF4E markers in histologically negative surgical margins, and their association with the clinical outcome of patients with head and neck squamous cell carcinoma
}

\author{
JAGTAR SINGH $^{1}$, RAMA JAYARAJ ${ }^{1}$, SIDDHARTHA BAXI ${ }^{2}$, MARIANA MILEVA ${ }^{3}$, \\ JOHN SKINNER $^{3}$, NAVNEET K. DHAND ${ }^{4}$ and MAHIBAN THOMAS ${ }^{5}$ \\ ${ }^{1}$ School of Psychological and Clinical Sciences, Charles Darwin University, Darwin, NT 0815; \\ ${ }^{2}$ Genesis Health Care Bunbury, Bunbury, WA 6230; ${ }^{3}$ Department of Anatomical Pathology, Royal Darwin Hospital, \\ Darwin, NT 0810; ${ }^{4}$ Faculty of Veterinary Science, University of Sydney, Camden, NSW 2570; \\ ${ }^{5}$ Department of Head and Neck Surgery, Royal Darwin Hospital, Darwin, NT 0811, Australia
}

Received August 28, 2015; Accepted October 28, 2015

DOI: $10.3892 / \mathrm{mco} .2015 .689$

\begin{abstract}
Molecular markers can be used to identify residual cancer at the surgical margins of head and neck squamous cell carcinoma (HNSCC) and assist in evaluating the complete resection of the tumour. The purpose of the present study was to investigate the expression levels of prognostic molecular markers at the histological tumour free surgical margins. In the present clinical retrospective study, 24/48 patients were selected with negative surgical margins for further analysis with immunohistochemical staining. Contingency tables and Fisher's exact tests were used to investigate the association between the expression levels of p53 and eukaryotic translation imitation factor 4E (eIF4E) with the clinical outcomes for patients with HNSCC. The expression levels of p53 and eIF4E were 54.2 and $87.5 \%$, respectively, in the surgical margins of patients with HNSCC. A total of 3/7 patients with recurrent cancer $(42.8 \%)$ were identified with p53-positive margins, and $6(85.7 \%)$ patients exhibited recurrence with eIF4e-positive margins. No statistically significant differences were identified for the recurrence risk between the overexpression of p53 and eIF4E in the surgical margins $(\mathrm{P}=0.88$ and $\mathrm{P}=0.99$, respectively). The eIF4E marker appears to be a more marked prognosticator compared with $\mathrm{p} 53$, as overexpression of eIF4E was identified in the margins of $6 / 7$ patients with local recurrence.
\end{abstract}

Correspondence to: Mr. Mahiban Thomas, Department of Head and Neck Surgery, Royal Darwin Hospital, 105 Rocklands Drive, Tiwi, Darwin, NT 0811, Australia

E-mail: Mahiban.Thomas@nt.gov.au

Key words: eukaryotic translation imitation factor 4E, immunohistochemistry, molecular markers, p53, recurrence, overall survival

\section{Introduction}

The successful treatment of patients with head and neck squamous cell carcinoma (HNSCC) depends on the early detection and the appropriate therapy (1). The incomplete resection of the primary tumour frequently leads to a decrease in the survival rate as a consequence of increased risk of recurrence; therefore, complete surgical resection of the primary tumour is a crucial prognostic step for $\operatorname{HNSCC}(2,3)$.

Molecular markers can be used to establish tumour free surgical margins and assist in the complete resection of the tumour (4). The molecular marker, p53, has been established to predict for recurrence in the surgical margins $(5,6)$. Mutation of the p53 gene leads to the pathogenesis of patients with HNSCC exhibiting expression levels ranging between 50 and $60 \%$ of the tumour cells $(7,8)$. A previous study suggests that immunohistochemical (IHC) staining with p53 has an advantage over histopathology to identify the patients at high risk for local recurrence (6). IHC analysis of the expression of p53 may therefore be a diagnostic of margin status and prognostic in the clinical management of HNSCC (9).

Overexpression of eukaryotic translation imitation factor 4E (eIF4E) is involved in the initiation of protein synthesis (10). However, overexpression of eIF4E not only induces the transformation and tumorigenesis, but also initiates metastasis (10). Previous studies have observed 100\% expression of eIF4E in HNSCC $(11,12)$. Clinically, the overexpression of eIF4E is commonly observed in a variety of human tumour types, and its overexpression is usually associated with disease progression, higher tumour recurrence rate and tumour-associated mortality $(13,14)$. In previous studies of breast and bladder cancer, the overexpression of eIF4E protein also correlated with an increased risk of disease progression and poor prognosis $(10,15,16)$. Another previous study reported that the overexpression of eIF4E in tumour free surgical margins correlated with local recurrence in patients with HNSCC (17). 
The 5-year survival rate for HNSCC remains low at $\sim 50 \%(18,19)$. A five year survival rate for HNSCC is significantly lower compared with other cancer types, including colorectal, cervix and breast $(18,20)$. With the advent of novel surgical procedures, improved radiotherapy and concomitant chemotherapy, there is a considerable improvement in the local rates (6). By contrast, the survival rates for HNSCC have failed to significantly improve $(21,22)$ since it is generally believed that incomplete resection of the primary tumour is the principal reason of local recurrence and mortality from HNSCC (2).

The present retrospective clinical study aimed to investigate the prognostic significance of the molecular markers, p53 and eIF4E, in the histologically tumour free surgical margins of HNSCC. In addition, the present study was designed to analyse the association between the expression levels of the p53 and eIF4E molecular markers with the clinical outcomes, including recurrence and survival, and also assessed whether eIF4E is more sensitive compared with p53 in predicting for the risk of recurrence.

\section{Materials and methods}

Study design. The present retrospective clinical study was performed on patients who underwent primary surgical resection for HNSCC. A total of 48 patients with HNSCC diagnosed at the Royal Darwin Hospital between 2006 and 2009 were identified. Out of the 48 patients, 24 were selected based on hematoxylin and eosin (H\&E) staining, and hospital records. The present study was approved by the Human Research Ethics Committee of the Northern Territory, Department of Health and Menzies School of Health Research (HR-10-1490).

Selection criteria. The inclusion criteria were as follows: i) The patient was treated at the Royal Darwin Hospital throughout their management; ii) The patients underwent surgical resection of a mucosal oropharyngeal cancer at Royal Darwin Hospital between 2006 and 2009; iii) the hospital reports for the patients were available; iv) the patient slides were available. The exclusion criteria were as follows: i) Patients with nodal diseases; ii) patients who were lost at follow-up.

Specimen collection. Paraffin-embedded tissue blocks from the surgical margins were obtained following the surgical resection and final pathology reports showing that the blocks were histologically free of tumour. In the present clinical retrospective study, a pathologist reviewed all the H\&E stained sections to confirm tumour-free surgical margins. Another two $5 \mu \mathrm{m}$ thick sections were mounted onto poly-L-lysine-coated slides for IHC staining with mouse monoclonal anti-human anti-p53 (cat.no. M7001; 1:200; Dako Australia Pty, Ltd., Campbellfield, VIC, Australia) and rabbit polyclonal anti-human anti-eIF4E (cat. no. ab47482; 1:500; Abcam, Cambridge, MA, USA) antibodies. The details of the staining technique and analysis are described below.

Clinical data entry. The anatomical pathology department based at the Royal Darwin Hospital coded each specimen at the time of acquisition and the specimen was tracked using that code. A computerised notebook linked the code numbers to the patient data and hospital records. In the present study, the pathologists scored the tumours in the surgical margins in a blinded manner. In addition, data entry was coded and no inadvertent bias was presented to the physician during patient follow-up.

IHC staining. The Ventana Benchmark XT machine (Ventana Medical Systems, Inc., Tucson, AZ, USA) has a programmed p53 and eIF4E IHC procedure file for processing the tissue samples. Each procedure file consisted of a specific sequence for buffer rinse, enzyme inhibitors, blocking serum, antibody detection complexes, chromogens and counter-stains. These reagents were used according to the manufacturer's instructions (Ventana Medical Systems, Inc.).

Following a series of buffer rinses and normal serum pre-incubation, $100 \mu 1$ monoclonal mouse p53 antibody (Dako Pty Ltd., Campbellfield, VIC, Australia) at a dilution of 1:200 and polyclonal rabbit eIF4E (Abcam, Cambridge, MA, USA) antibody at a dilution of 1:500 were dispensed per slide. The slides were treated with streptavidin-enzyme conjugate with diaminobenzidine (Dako Pty Ltd.).

At the end of the automated staining process, the slides were washed in hot water with detergent for $3 \mathrm{~min}$ to remove the oily material and were subsequently dehydrated through a series of ethanol dilutions, followed by treatment in xylene. Finally, cover slips were placed on each slide and fixed with the permanent mounting material.

Microscopic examination. All immunohistochemically stained slides were assessed for the expression levels of p53 and eIF4E protein by light microscopy by two clinical pathologists in a blinded manner. The stained slides of tumour margins were evaluated for the protein expression levels of p53 and eIF4E. The 5\% cut-off value was selected on the basis of the expression levels of p53 and eIF4E in tumour cells in the basal cell layer of surgical margin (7). Therefore, surgical margins were considered as positive if IHC staining was observed in $>5 \%$ of the cells in the epithelial layer.

These tumour margins were considered as positively expressing p53 if there was a brown nuclear staining in the basal cell layer of epithelium. Similarly, surgical margins were considered as positively expressing eIF4E if there was a reddish brown peri-nuclear cytoplasmic staining of $>5 \%$ in the layer of epithelium.

Statistical analysis. The clinical characteristics of patients with HNSCC and surgical margins were examined statistically using SAS version 9.3 software (SAS Institute Inc., Cary, NC, USA). The time prior to recurrence was considered as the period from surgery until the date of the first documented recurrence among the patients (7). Local recurrence was defined as a recurrence in the original tumour bed (23). The overall survival time was defined as the interval between the dates of the beginning of the treatment (surgery) and mortality, or the last information for censored observations (24).

Contingency tables and Fisher's exact tests were used to evaluate the association of p53 and eIF4E in the surgical margins with age, sex, race, anatomical site, tumour stage, nodal status, nodal stage, post-operative therapy and histological grade. The recurrence and survival rate were estimated 
Table I. Clinical characteristics of 24 patients, according to the expression levels of p53 and eIF4E on tumour margin.

\begin{tabular}{|c|c|c|c|c|c|c|}
\hline \multirow[b]{2}{*}{ Clinical characteristic } & \multicolumn{2}{|c|}{$\mathrm{p} 53$} & \multirow[b]{2}{*}{ P-value } & \multicolumn{2}{|c|}{ eIF4E } & \multirow[b]{2}{*}{ P-value } \\
\hline & Negative & Positive & & Negative & Positive & \\
\hline Age, Mean, years & 60 & & & 60 & & 0.2 \\
\hline \multicolumn{7}{|l|}{ Gender } \\
\hline Female & 1 & 2 & & 0 & 3 & \\
\hline Male & 3 & 1 & 0.64 & 3 & 18 & 1.0 \\
\hline \multicolumn{7}{|l|}{ Race } \\
\hline Indigenous & 2 & 2 & & 0 & 4 & \\
\hline Non-Indigenous & 9 & 11 & 0.85 & 3 & 17 & 1.0 \\
\hline \multicolumn{7}{|l|}{ Anatomical sites } \\
\hline Floor of mouth & 8 & 6 & & 1 & 13 & \\
\hline Tongue & 1 & 2 & & 1 & 2 & \\
\hline Lips & 2 & 3 & & 1 & 4 & \\
\hline Tonsil & 0 & 2 & 0.6 & 0 & 2 & 0.46 \\
\hline \multicolumn{7}{|l|}{ Tumour stage } \\
\hline $\mathrm{T} 1$ & 4 & 7 & & 1 & 10 & \\
\hline $\mathrm{T} 2$ & 3 & 3 & & 1 & 5 & \\
\hline T3 & 0 & 0 & & 0 & 0 & \\
\hline $\mathrm{T} 4$ & 4 & 3 & 0.67 & 1 & 6 & 0.88 \\
\hline \multicolumn{7}{|l|}{ Nodal status } \\
\hline Negative & 8 & 10 & & 3 & 15 & \\
\hline Positive & 3 & 3 & 0.81 & 0 & 6 & 0.54 \\
\hline \multicolumn{7}{|l|}{ Nodal stage } \\
\hline No & 8 & 10 & & 3 & 15 & \\
\hline N1 & 2 & 3 & & 0 & 5 & \\
\hline $\mathrm{N} 2 \mathrm{~b}$ & 1 & 0 & 0.81 & 0 & 1 & 1.0 \\
\hline \multicolumn{7}{|l|}{ Post-op XRT } \\
\hline No & 5 & 3 & & 2 & 6 & \\
\hline Yes & 6 & 10 & 0.25 & 1 & 15 & 0.2 \\
\hline \multicolumn{7}{|l|}{ Histological grade } \\
\hline Poorly differentiated & 3 & 1 & & 1 & 3 & \\
\hline Moderate differentiated & 2 & 4 & 1 & 5 & & \\
\hline Well differentiated & 7 & 7 & 0.61 & 1 & 13 & 0.5 \\
\hline
\end{tabular}

P-value obtained by Fisher's exact tests. T1, Tumour $\leq 2 \mathrm{~cm}$; T2, $>2 \mathrm{~cm}$ and $<4 \mathrm{~cm} ; \mathrm{T} 3,>4 \mathrm{~cm}$, T4, Tumour with extension to bone, muscle, skin, antrum or neck; XRT, irradiation therapy.

by the Kaplan-Meier method and compared using the log-rank test $(7,25)$.

\section{Results}

Clinical characteristics of the patients. The clinical characteristic records of the 24 patients with HNSCC were obtained from the Royal Darwin Hospital and the Department of Births, Deaths and Marriages (Darwin, Australia). Those records were reviewed to assess patient survival. The clinical characteristics included age, sex, race, anatomical site, tumour stage, nodal status, nodal stage, post-operative radiation therapy and histological grade. The median age was 60 years (range, 46-81 years). A total of 21 patients were male and three were female; 4 were indigenous and 20 were non-indigenous. The median follow-up period was 74 months (range, 1-74 months). Of the 24 patients, 12 patients succumbed to mortality and 12 patients remained alive at the end of the present study. The floor of the mouth was the most common anatomical sub-site $(14 / 24 ; 58.3 \%)$, followed by the tongue $(5 / 24 ; 20.8 \%)$, lips $(3 / 24 ; 12.5 \%)$ and tonsil $(2 / 24 ; 8.4 \%)$. No statistically significant association was observed between the expression of p53 and eIF4E, and the clinical characteristics of patients with HNSCC (Table I).

IHC analysis for p53 and eIF4E. Of the 24 patients with histologically negative margins, $13(54.2 \%)$ patients exhibited p53-positive and $21(87.5 \%)$ patients exhibited eIF4e-positive, with at least one of the tumour margins positively stained. Fig. 1 demonstrated an HNSCC primary tumour and surgical 
Table II. Local recurrence of 24 patients, according to the expression levels of p53 and eIF4E on tumour margin.

\begin{tabular}{|c|c|c|c|c|c|c|}
\hline \multirow[b]{2}{*}{ Local recurrence } & \multicolumn{2}{|c|}{ p53 } & \multirow[b]{2}{*}{ P-value } & \multicolumn{2}{|c|}{ eIF4E } & \multirow[b]{2}{*}{ P-value } \\
\hline & Negative & Positive & & Negative & Positive & \\
\hline Non-recurrence & 10 & 7 & & 15 & 2 & \\
\hline Recurrence & 3 & 4 & 0.88 & 6 & 1 & 0.99 \\
\hline
\end{tabular}

Table III. Recurrence and survival of head and neck squamous cell carcinoma patients according to the p53 and eIF4E status of surgical margin.

\begin{tabular}{|c|c|c|c|c|c|}
\hline \multirow[b]{2}{*}{ Clinical outcomes } & \multicolumn{2}{|c|}{ p53 expression } & \multicolumn{2}{|c|}{ eIF4E expression } & \multirow[b]{2}{*}{ p53/eIF4e-positive (\%) } \\
\hline & Positive (\%) & Negative (\%) & Positive (\%) & Negative (\%) & \\
\hline \multicolumn{6}{|l|}{ Recurrence } \\
\hline Alive & $1(14.3)$ & $2(28.6)$ & $3(42.8)$ & $0(0)$ & $1(4.2)$ \\
\hline Died & $2(28.6)$ & $2(28.6)$ & $3(42.8)$ & $1(14.1)$ & $2(8.3)$ \\
\hline \multicolumn{6}{|l|}{ No recurrence } \\
\hline Alive & $5(29.4)$ & $4(23.5)$ & $8(47.1)$ & $1(5.8)$ & $5(20.8)$ \\
\hline Died & $5(29.4)$ & $3(17.7)$ & $7(41.2)$ & $1(5.9)$ & $5(20.8)$ \\
\hline
\end{tabular}

margins stained with H\&E, p53 and eIF4E markers, respectively. The sections of solid tumour stained with H\&E showed cords and nests of malignant squamous epithelial cells exhibiting nuclear pleomorphism, hyperchromatism and keratin pearls (Fig. 1A). The identical tumour section was also stained for p53 and exhibited dark brown staining of the nucleus of the tumour cells (Fig. 1B). In addition, the identical tissue section was also stained with eIF4E antibody and exhibited brown peri-nuclear cytoplasmic staining of the tumour cells in the epithelial layer (Fig. 1C).

Furthermore, Fig. 1D and E illustrated negative surgical margins with $\mathrm{H} \& \mathrm{E}$ staining and $\mathrm{p} 53 \mathrm{IHC}$ staining, respectively. However, the identical tumour margin exhibited positive staining for eIF4E (brown peri-nuclear cytoplasmic) around the residual cancer cells in the epithelial layer (Fig. 1F).

Association of p53 and eIF4E overexpression with clinical outcomes. Of the 24 patients with HNSCC, 7 (29.2\%) patients exhibited tumour recurrence and 12 (50\%) patients succumbed to mortality during the follow-up period. For those patients who exhibited p53-positive staining, 3/13 patients (23.1\%) had local recurrence and 10 (76.9\%) showed no recurrence. In the identical way, the patients who were eIF4e-positive, 6/21 patients (28.5\%) had a recurrence and 15 (71.5\%) showed no recurrence. In addition, 10/11 (90.9\%) patients with p53-negative margins and $2 / 3$ patients $(66.6 \%)$ with eIF4e-negative margins had no local recurrence. However, one patient with a recurrence exhibited a p53- and eIF4e-negative surgical margin. No significant difference was observed with regards to local recurrence for p53-positive and p53-negative patients ( $\mathrm{P}=0.88)$. Similarly, no statistical significance was observed in the local recurrence in patients exhibiting eIF4e-positive or eIF4e-negative surgical margins ( $\mathrm{P}=0.99$; Table II).
A total of $12 / 24$ patients (50\%) during the follow-up period and 7/13 p53-positive patients (53.8\%) succumbed to mortality . It was shown that $6(46.2 \%)$ patients who survived exhibited positive p53 expression in their surgical margins. Of the 21 patients with eIF4e-positive margins, 10 patients succumbed to mortality and 11 remained alive. No significant association was observed between the overall survival, and $\mathrm{p} 53$-positive and negative surgical margins $(\mathrm{P}=0.36)$. Similarly, no association was identified between eIF4e-positive and negative margins, and the overall survival of patients with $\operatorname{HNSCC}(\mathrm{P}=0.64)$.

Table III compared the comparative study of recurrence and survival of patients with HNSCC, with p53 and eIF4E status of surgical margins. The patients were divided into two groups, the recurrence group and non-recurrence group. In the recurrence group, one (4.2\%) patient survived with positive p53 and eIF4E surgical margins, and two (8.3\%) patients exhibiting the identical pattern succumbed to mortality. In the non-recurrence group, 5 (20.8\%) patients survived with positive p53 and eIF4E surgical margins; whereas, 5 (20.8\%) patients succumbed to mortality during the follow-up period. Fig. 2 demonstrated the Kaplan-Meier curve with three combinations of p53 and eIF4E expression, including p53-positive and eIF4E-negative, p53-positive and eIF4E-negative, and p53-positive and eIF4E-positive. No significant differences were observed between these three different combinations $(\mathrm{P}=0.46)$.

\section{Discussion}

Immunodiagnostic investigation of the overexpression of p53 and eIF4E in the surgical margin may contribute to early detection of residual disease. The patients with a tumour received 

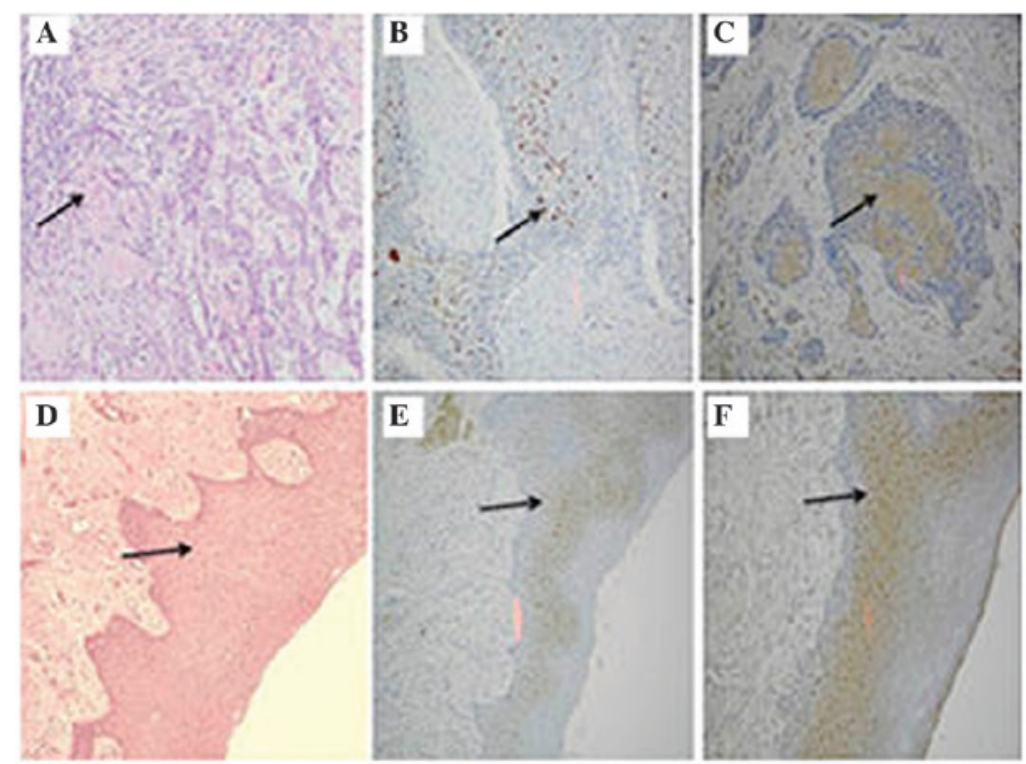

Figure 1. Comparison different staining in the primary tumour and histological tumour free surgical margins of HNSCC. (A) HNSCC cells were stained with H\&E. (B) HNSCC cells with immunohistochemically stained for p53. Positive staining was an intense brown nuclear staining of p53. (C) HNSCC cells with immunohistochemically stained for eIF4E. Positive staining was an intense reddish brown peri-nuclear cytoplasmic staining of eIF4E. (D) Histological tumour free surgical margin with mild dysplasia was observed following H\&E staining. (E) The identical margin showed negative staining in the basal cell layer with p53. (F) Section D showed reddish peri-nuclear cytoplasmic staining of the epithelial cells with eIF4E (magnification, x20). HNSCC, head and neck squamous cell carcinoma; H\&E, heamatoxylin and eosin.

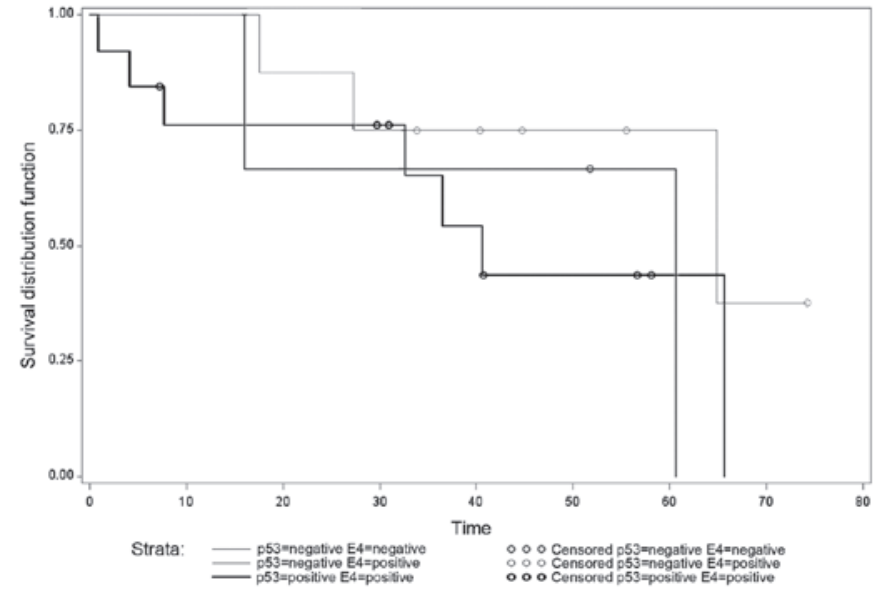

Figure 2. Kaplan-Meier recurrence curves with the expression levels of p53 and eIF4E in surgical margins. Kaplan-Meier curves comparing the combined effects of the expression levels of p53 and eIF4E in the surgical margins against the expression of each marker alone. These curves estimate the recurrence rate of patients with head and neck squamous cell carcinoma and revealed no significant difference in the probability of having no recurrence according the p53 and eIF4E expression status in surgical margins.

a more direct and effective treatment therapy to improve the survival rate. The results from Nathan et al (7) showed the impact of the overexpression of p53 and eIF4E in the recurrence and survival in HNSCC. The overexpression of eIF4E is more consistent compared with p53 HNSCC (4). Therefore, eIF4E is often considered as a specific tumour marker for HNSCC, and is more sensitive compared with p53 (26).

The overexpression of eIF4E is more common in HNSCC compared with p53 and it occurs earlier in the process of tumorigenesis (7). Mutations of p53 in HNSCC are not only a common event, but also occur comparatively late in tumour progression (7). In patients with HNSCC, p53 expression has been observed as a late event in the carcinogenesis (27). Therefore, mutations in p53 are less common in pre-invasive cancer compared with in invasive cancer (28). Li et al (26) reported that the overexpression of p53 was observed in $63 / 112$ patients $(56.3 \%)$, while that of eIF4E was observed in $105(93.8 \%)$. Nathan et al (13) identified 22/54 patients (42\%) with p53-positive and 27 (52\%) with eIF4E-positive staining in the surgical margins of patients with HNSCC. In the present retrospective study, the overexpression of p53 was identified in $13 / 24$ patients $(54.2 \%)$ and overexpression of eIF4E was identified in 21 patients $(87.5 \%)$ with HNSCC. A lower frequency of p53 expression in surgical margins may be attributed to the small sample size in the present study.

Overexpression of eIF4E in the primary tumours was not a significant predictor of recurrence (7). It appeared that the majority of solid tumours require an elevated expression of eIF4E for tumour progression. However, p53 and eIF4E expression in surgical margins were often associated with an increase in recurrence rate (7). Nathan et al (13) reported that, 14/52 patients exhibited recurrence and a significant difference was observed in the recurrence rate between eIF4E-positive and eIF4E-negative margins $(\mathrm{P}=0.003)$. This previous study reported that $12 / 14$ recurrent patients $(86 \%)$ were identified with eIF4e-positive margins. However, no significant difference was observed between p53-positive and p53-negative margin recurrence rate $(\mathrm{P}=0.11)(13)$. A previous study showed that $33.3 \%(9 / 27)$ of recurrent patients were p53-positive margins and eIF4E-positive was 63.6\% (14/27), demonstrating a significant difference in the HNSCC margin $(\mathrm{P}=0.006)(16)$.

In the present retrospective study, 6/7 cancer recurrent patients $(85.7 \%)$ had eIF4e-positive margins, whereas only 
three patients had recurrence $(42.8 \%)$ with p53-positive margins. All three recurrent patients with p53 overexpression also exhibited eIF4E overexpression in the surgical margins. In addition, these results revealed no significant association between the expression levels of p53 and eIF4E with local recurrence $(\mathrm{P}=0.46)$. The small numbers of patients with HNSCC in the present study may not be sufficient to demonstrated the recurrence risk with respect to expression of p53 and eIF4E on surgical margins.

Liangping et al (16) reported that the 5 year survival rate in the p53-positive margin (13/67 cases) and p53-negative margin group (54/67 cases) was 24.62 and $75.69 \%$ respectively, with the difference being significant $(\mathrm{P}=0.0012)$. In that same study, the 5-year survival rate with the eIF4e-positive group (22 cases) and eIF4e-negative group (45 cases) was 43.31 and $77.52 \%$, respectively, and the results showed significant differences $(\mathrm{P}=0.0006)$.

In the present study, $10 / 12$ patients (83.3\%) with eIF4e-positive surgical margins, and seven patients (53.3\%) with p53-positive margins succumbed to mortality. All 7 patients who succumbed to mortality with p53-positive margins also exhibited eIF4e-positive surgical margins. Statistically, no significant difference was observed when the p53-positive and p53-negative patients were compared for overall survival $(\mathrm{P}=0.36)$. Similarly, no significant differences were observed between eIF4e-positive and eIF4e-negative patients with HNSCC $(\mathrm{P}=0.64)$. Therefore, the present study observed that patients with eIF4E overexpression had a higher mortality rate compared with patients with p53 overexpression, although this difference was not statistically significant.

In the present study, non-significant results were obtained regarding the association between p53 and eIF4E overexpression, and the clinical outcomes of the 24 HNSCC patients. However, the expression of eIF4E appears to be a more marked prognosticator compared with p53 since the overexpression of eIF4E was observed in the margins of 6/7 patients who had local recurrence and p53 was observed in only three patients. Therefore, a prospective study in the near future with a larger number of patients with HNSCC would be ideal to analyse the sensitivity and specificity of eIF4E in the management of HNSCC and to unmask the contribution of overexpression in association with recurrence and overall survival.

\section{Acknowledgements}

The present clinical study was supported by the School of Psychological and Clinical Sciences, Charles Darwin University, Australia. We also want to thank the clinical pathologists based at the Anatomical Pathology Department at Royal Darwin Hospital for their assistance, which was vital for the IHC work in the present study.

\section{References}

1. Rezende TM, de Souza Freire M and Franco OL: Head and neck cancer: Proteomic advances and biomarker achievements. Cancer 116: 4914-4925, 2010.

2. Armstrong W, Vokes DE and Maisel RH: Malignant tumors of the larynx, cummings otolaryngology head \& neck surgery, 5th edn. America: Mosby Elsevier. 482-511, 2010.
3. Jalali MM, Heidarzadeh A, Zavarei MJ and Sarmast H: p53 overexpression impacts on the prognosis of laryngeal squamous cell carcinomas. Asian Pac J Cancer Prev 12: 1731-1734, 2011

4. Nathan CO, Franklin S, Abreo FW, Nassar R, De Benedetti A and Glass J: Analysis of surgical margins with the molecular marker eIF4E: A prognostic factor in patients with head and neck cancer. J Clin Oncol 17: 2909-2914, 1999.

5. Breda A, Konijeti R and Lam JS: Patterns of recurrence and surveillance strategies for renal cell carcinoma following surgical resection. Expert Rev Anticancer Ther 7: 847-862, 2007.

6. Van Houten VM, Leemans CR, Kummer JA, Dijkstra J, Kuik DJ, van den Brekel MW, Snow GB and Brakenhoff RH: Molecular diagnosis of surgical margins and local recurrence in head and neck cancer patients: A prospective study. Clin Cancer Res 10: 3614-3620, 2004.

7. Nathan CO, Sanders K, Abreo FW, Nassar R and Glass J: Correlation of p53 and the proto-oncogene eIF4E in larynx cancers: Prognostic implications. Cancer Res 60: 3599-3604, 2000 .

8. Bradford CR, Kumar B, Bellile E, Lee J, Taylor J, D'Silva N, Cordell K, Kleer C, Kupfer R, Kumar P, et al: Biomarkers in advanced larynx cancer. Laryngoscope 124: 179-187, 2014.

9. Gasco M and Crook T: The p53 network in head and neck cancer. Oral Oncol 39: 222-231, 2003.

10. De Benedetti A and Graff JR: eIF-4E expression and its role in malignancies and metastases. Oncogene 23: 3189-3199, 2004.

11. Cardesa A and Nadal A: Carcinoma of the head and neck in the HPV era. Acta Dermatovenerol Alp Pannonica Adriat 20: 161-173, 2011.

12. Nathan CO, Liu L, Li B, Abreo FW, Nandy I and De Benedetti A: Detection of the proto-oncogene eIF4E in surgical margins may predict recurrence in head and neck cancer. Oncogene 15: 579-584, 1997.

13. Nathan CO, Amirghahri N, Rice C, Abreo FW, Shi R and Stucker FJ: Molecular analysis of surgical margins in head and neck squamous cell carcinoma patients. Laryngoscope 112: 2129-2140, 2002

14. Salehi Z and Mashayekhi F: Expression of the eukaryotic translation initiation factor 4E (eIF4E) and 4E-BP1 in esophageal cancer. Clin Biochem 39: 404-409, 2006.

15. Crew JP, Fuggle S, Bicknell R, Cranston DW, de Benedetti A and Harris AL: Eukaryotic initiation factor-4E in superficial and muscle invasive bladder cancer and its correlation with vascular endothelial growth factor expression and tumour progression. Br J Cancer 82: 161-166, 2000.

16. Liangping $X$ : The prognostic value of pathological and molecular margins marked by p53 and elF4E in laryngeal carcinoma. Chinese-German J Clin Oncol 4: 56-60, 2005.

17. Chakraborty S, Mohiyuddin SM, Gopinath KS and Kumar A: Involvement of TSC genes and differential expression of other members of the mTOR signaling pathway in oral squamous cell carcinoma. BMC Cancer 8: 163, 2008.

18. Jemal A, Siegel R, Ward E, Hao Y, Xu J, Murray T and Thun MJ: Cancer statistics, 2008. CA Cancer J Clin 58: 71-96, 2008.

19. Kokko LL, Hurme S, Maula SM, Alanen K, Grénman R, Kinnunen I and Ventelä S: Significance of site-specific prognosis of cancer stem cell marker CD44 in head and neck squamous-cell carcinoma. Oral Oncol 47: 510-516, 2011.

20. Clark C, Shah S, Herman-Ferdinandez L, Ekshyyan O, Abreo F, Rong X, McLarty J,Lurie A, Milligan EJ and Nathan CO: Teasing out the best molecular marker in the AKT/mTOR pathway in head and neck squamous cell cancer patients. Laryngoscope 120: 1159-1165, 2010.

21. Partridge M, Costea DE and Huang X: The changing face of p53 in head and neck cancer. Int J Oral Maxillofac Surg 36: 1123-1138, 2007.

22. Argiris A, Karamouzis MV, Raben D and Ferris RL: Head and neck cancer. Lancet 371: 1695-1709, 2008.

23. Chao YK, Chuang WY, Yeh CJ, Chang YS, Wu YC, Kuo SY, Hsieh MJ and Hsueh C: High phosphorylated 4E-binding protein 1 expression after chemoradiotherapy is a predictor for locoregional recurrence and worse survival in esophageal squamous cell carcinoma patients. J Surg Oncol 105: 288-292, 2012.

24. Waitzberg AF, Nonogaki S, Nishimoto IN, Kowalski LP, Miguel RE, Brentani RR and Brentani MM: Clinical significance of c-myc and p53 expression in head and neck squamous cell carcinomas. Cancer Detect Prev 28: 178-186, 2004. 
25. Song HS, Do YR, Kang SH, Jeong KY and Kim YS: Prognostic significance of immunohistochemical expression of p53 gene product in operable breast cancer. Cancer Res Treat 38: 218-223, 2006.

26. Li S and Wei Q: The expression and the clinical significance of eukaryotic translation initiation factors $4 \mathrm{E}$ and p53 in squamous cell carcinoma. Chinese-German J Clin Oncol 8: 286-288, 2009.
27. Boyle JO, Hakim J, Koch W, van der Riet P, Hruban RH, Roa RA, Correo R, Eby YJ, Ruppert JM and Sidransky D: The incidence of p53 mutations increases with progression of head and neck cancer. Cancer Res 53: 4477-4480, 1993.

28. Pihan GA, Wallace J, Zhou Y and Doxsey SJ: Centrosome abnormalities and chromosome instability occur together in pre-invasive carcinomas. Cancer Res 63: 1398-1404, 2003. 